Article

\title{
Optimal Incentives in a Principal-Agent Model with Endogenous Technology
}

\author{
Marco A. Marini ${ }^{1}$, Paolo Polidori ${ }^{2}$, Désirée Teobaldelli ${ }^{2}$ and Davide Ticchi ${ }^{3, *}$ \\ 1 Department of Social and Economic Sciences, Sapienza University of Rome; Piazzale Aldo Moro 5, \\ 00185 Rome, Italy; marini@dis.uniroma1.it \\ 2 Department of Law, University of Urbino, Via Matteotti 1, 61029 Urbino, Italy; \\ paolo.polidori@uniurb.it (P.P.); desiree.teobaldelli@uniurb.it (D.T.) \\ 3 Department of Economics and Social Sciences, Marche Polytechnic University, Piazzale Martelli 8, \\ 60121 Ancona, Italy \\ * Correspondence: d.ticchi@univpm.it; Tel.: +39-071-220-7086; Fax: +39-071-220-7102
}

Received: 2 November 2017; Accepted: 26 January 2018; Published: 5 February 2018

\begin{abstract}
One of the standard predictions of the agency theory is that more incentives can be given to agents with lower risk aversion. In this paper, we show that this relationship may be absent or reversed when the technology is endogenous and projects with a higher efficiency are also riskier. Using a modified version of the Holmstrom and Milgrom's framework, we obtain that lower agent's risk aversion unambiguously leads to higher incentives when the technology function linking efficiency and riskiness is elastic, while the risk aversion-incentive relationship can be positive when this function is rigid.
\end{abstract}

Keywords: principal-agent; incentives; risk aversion; endogenous technology

JEL Classification: D21; D81; D82; D86; M52; O33

\section{Introduction}

One of the main results of the agency theory is the trade-off between incentives and insurance. Lower agent's risk aversion allows the principal to provide more incentives by making the payment of the agent more related to output, while higher uncertainty increases the gains from insuring the agent and reduces the pay-for-performance sensitivity (e.g., Holmstrom and Milgrom [1] ). However, the empirical works testing the link between uncertainty and incentives have found mixing results (e.g., Rao and Hanumantha [2], Allen and Lueck [3], Aggarwal and Samwick [4], Core and Guay [5], and Wulf [6]). In many cases, the empirical findings are even in contradiction with the standard predictions of the theory as they document a positive (rather than negative) correlation between observed measures of uncertainty and the provision of incentives (see Prendergast [7] for an extensive discussion on this point).

Some recent contributions on the matching literature (e.g., Wright [8], Legros and Newman [9], Serfes [10,11], and Li and Ueda [12]) has attempted to offer a theoretical justification of the above cited results. The explanation provided has been based on the endogenous matching between principals and agents by introducing the heterogeneity on managers' degree of risk aversion (Wright [8]), on the assortative matching between risk-averse agents and riskier principals (Serfes [10]), or on the matching between agents and firms that differ, respectively, for the level of productivity and riskiness ( $\mathrm{Li}$ and Ueda [12]).

Differently from the logic developed in the matching models, in this paper, we propose an alternative and simpler explanation of the relationship between risk and incentives based on the endogeneity of the technology adopted by the principal. In particular, we show that the traditional 
relationship between agent's risk aversion and optimal incentive may be absent or reversed when the technology is endogenous and projects with higher efficiency are also riskier.

To describe the mechanisms at work in our theory, we propose a modified version of the Holmstrom and Milgrom's [1] framework, where the principal can choose among a continuous set of projects where those with higher efficiency are also characterized by higher riskiness. While the employment of Holmstrom and Milgrom's framework implies some simplifying assumptions (such as constant absolute risk aversion utility function, quadratic costs and normally distributed shocks), it allows us to describe the mechanisms at work in a clear and simple model that generates closed-form solutions.

We obtain that lower agent's risk aversion unambiguously leads to higher incentives only when the technology function linking risk and efficiency is elastic, while the risk aversion-incentive relationship can be positive when this function is rigid. This is because a lower risk aversion of the agent makes it optimal for the principal the adoption of a riskier and a more efficient technology. While the higher efficiency of the new technology (as well as the lower agent's risk aversion) allows the principal to give more incentives to the agent, its higher riskiness makes the provision of incentives more costly which works in the direction of reducing the optimal degree of the pay-for-performance sensitivity.

In other words, whereas less risk averse agents would normally induce the principal to provide more incentives, they also lead her to choose more productive and riskier technologies. The higher efficiency of the new technology also pushes towards an increase of incentives but the higher riskiness works in the opposite direction making it optimal their reduction. The relative strength of this latter effect is related to the elasticity of technology function linking efficiency and riskiness, and it is higher the lower such elasticity. When this situation occurs, the relationship between the agent's degree of risk aversion and incentives becomes positive.

As we anticipated above, our work is related to the matching literature that addresses the issue of the link between uncertainty and incentives. In particular, by introducing competition for heterogeneous managers, who differ in their degrees of risk aversion, Wright [8] proves that a negative or positive relationship between agents' risk and incentives is possible and depends on the relative risk aversion of the managers and the riskiness of the environments. Serfes [10] shows that, whereas under efficient positive assortative matching (in which higher risk-averse agents are optimally matched with riskier principals) the traditional trade-off between risk and incentives holds, under efficient negative assortative matching (lower risk-averse agents are matched with riskier principals) this trade-off can fail to hold, in particular when matching curves are very steep. Li and Ueda [12] show, instead, that, if the agents differ only in their productivity, safer firms will offer high-powered incentives schemes, in this way capturing the higher productive workers at the endogenous matching. Legros and Newman [9] look in general at the sufficient conditions for monotone matching when the utility between partners is not fully transferable. In an application to principal-agent model, they show that when the agents' risk aversion does not decline too quickly, the agents with lower risk aversion (or higher wealth) are matched to principals with safer projects. ${ }^{1}$ It is worth noting that, differently from matching models, where both principals and agents can select endogenously their partners, in our model the principal only decides the technology to adopt (with its peculiar riskiness) and the bonus paid to the agent to incentivize his effort. Here, neither the principal nor the agent is allowed to select its preferred partner.

The paper is organized as follows. In Section 2, we describe the framework, and Section 3 provides the solution of the model. Section 4 presents the comparative statics analysis of the effect of a reduction

1 This could also explain the counterintuitive tendency of wealthier peasants to tend safer crops than poor peasants in medieval sharecropping (Ackerberg and Botticini [13]). A few other authors have contributed to this important relationship in principal-agent models. Among them, Mookherjee and Ray [14] who model an infinitely repeated interaction among principals and agents randomly matched at each period, Barros and Macho-Stadler [15]who look into a situation where several principals compete for an agent and Dam and Castrillo [16]who propose a model to analyze an economy with several principals and agents in order to characterize the set of stable outcomes. 
of the agent's risk aversion on incentives. Section 5 provides a discussion on our framework and on a modified version of it. Section 6 concludes.

\section{The Framework}

We consider a moral hazard model as in Holmstrom and Milgrom [1]. The principal decides the technology and is risk neutral. The agent is risk averse and has a constant absolute risk aversion (CARA) utility function $u$ with a coefficient of absolute risk aversion equal to $r$, i.e.,

$$
u=-\exp \{-r x\},
$$

where $x$ is the agent's payoff. Total output is equal to

$$
y=e+\varepsilon,
$$

where $e$ is the agent's action (e.g., effort) and $\varepsilon$ is an (unobservable) random variable normally distributed with zero mean and variance $\sigma^{2}$. The technology is characterized by quadratic costs, so that the agent's cost of action is

$$
c(e)=\frac{k}{2} e^{2},
$$

where $k$ is a constant representing the efficiency of the technology employed. Better technologies are characterized by a lower cost parameter $k$ and vice-versa. The agent's reservation utility is equal to $\delta$.

We here modify the Holmstrom and Milgrom's framework by assuming the existence of a given set of technologies (or projects) with different levels of efficiency and riskiness among which the principal can choose. In particular, we assume a trade-off between efficiency and riskiness so that technologies with a higher volatility $\sigma^{2}$ also have a lower marginal cost of effort, i.e.,

$$
k \equiv k\left(\sigma^{2}\right) \text { with } k^{\prime} \equiv \frac{d k}{d \sigma^{2}}<0,
$$

where $k>0$ for all $\sigma^{2} \in\left(0, \bar{\sigma}^{2}\right) \cdot{ }^{2}$ For simplicity, the function $k(\cdot)$ is assumed to be a continuous and differentiable in $\sigma^{2}$. As we shall see, the following conditions on the technology function ensure that an interior solution for the optimal technology always exists: (i) $k^{\prime \prime}$ large enough, (ii) $\lim _{\sigma^{2} \rightarrow 0}\left(-k^{\prime} / r k^{2}\right)>1$, and (iii) $\lim _{\sigma^{2} \rightarrow \bar{\sigma}^{2}} k^{\prime}=0$.

The timing of events in our framework is the following: (1) the principal decides the optimal technology and the agent's payment scheme; and (2) the agent optimally chooses the action.

In the next sections, we determine the choices of the principal and of the agent, and analyze the effects of a variation of the agents' risk aversion on the optimal payment scheme of the agent.

\section{The Equilibrium}

We solve the problem by determining the optimal payment scheme and the agent's action for a given technology. ${ }^{3}$ Then, we determine the optimal technology choice of the principal.

Holmstrom and Milgrom [1] show that a linear payment is optimal in the above framework, so that the agent's payment can be written as $s(y)=\beta y+\alpha$, where $\alpha$ and $\beta$ are constants optimally

2 As it will be clear next, if the technological relationship between efficiency and riskiness would be reversed, i.e., $k^{\prime}>0$, then the equilibrium outcome would be trivial as the optimal choice of the principal is always the more efficient and safer technology.

3 We here omit some details of the analysis as the complete description of the solution can be found in Holmstrom and Milgrom [1]. 
chosen by the principal that have to be determined. Taking into account Equations (1)-(3) and the distribution of the shock, the agent's expected utility is

$$
\mathbb{E}\{-\exp \{-r[s(y)-c(e)]\}\}=-\exp \left\{-r\left[\beta e+\alpha-(1 / 2) k e^{2}-(1 / 2) r \beta^{2} \sigma^{2}\right]\right\},
$$

and therefore his maximization problem can be written as

$$
\max _{e} \beta e+\alpha-(1 / 2) k e^{2}-(1 / 2) r \beta^{2} \sigma^{2} .
$$

The first order condition of this problem is

$$
\beta=e k
$$

Substituting this latter condition into Equation (5), and then setting the expression (representing the agent's certainty equivalent) equal to $\delta$ gives ${ }^{4}$

$$
\alpha=-(1 / 2) k e^{2}+(1 / 2) r \beta^{2} \sigma^{2}+\delta .
$$

Hence, the principal's maximization problem becomes

$$
\max _{e} \pi=\mathbb{E}[y-s(y)]=e-(1 / 2) k e^{2}-(1 / 2) r k^{2} e^{2} \sigma^{2}-\delta,
$$

which gives the following well-known second best solution for the agent's action ${ }^{5}$

$$
e^{*}=\frac{1}{k\left(1+r k \sigma^{2}\right)} .
$$

Using the fact that $\beta=e k$, it follows that the optimal share of output paid to the agent is

$$
\beta^{*}=\frac{1}{1+r k \sigma^{2}},
$$

and the optimal fixed payment is

$$
\alpha^{*}=\frac{-1+r k \sigma^{2}}{2 k\left(1+r k \sigma^{2}\right)^{2}}+\delta .
$$

Let now $\sigma_{*}^{2}$ denote the variance of the optimal project. This is the solution of the following maximization problem of the principal

$$
\max _{\sigma^{2}} \pi^{*}=\frac{1}{2 k\left(1+r k \sigma^{2}\right)}-\delta,
$$

subject to the technological constraint in Equation (4). ${ }^{6}$

The first order condition of this problem is

$$
\frac{d \pi^{*}}{d \sigma^{2}}=-\frac{k^{\prime}+2 r k k^{\prime} \sigma^{2}+r k^{2}}{2 k^{2}\left(1+r k \sigma^{2}\right)^{2}}=0,
$$

4 It is worth remarking that in this framework the principal own the technology and, therefore, she will always offer a payment scheme giving the agent an expected utility equal to his reservation utility (i.e., the agent's certainty equivalent) $\delta$.

5 The first order condition of the problem in Equation (6) is $d \pi / d e=1-k e-r k^{2} e \sigma^{2}=0$ and the second order condition is always satisfied as $d^{2} \pi / d e^{2}=-k-r k^{2} \sigma^{2}<0$.

6 It is immediate that the maximized expected profit $\pi^{*}$ (for a given technology) is obtained from the substitution of Equation (7) into Equation (6). 
and, therefore, the variance $\sigma_{*}^{2}$ of the optimal project is implicitly defined by the following equation:

$$
F\left(\sigma^{2}\right) \equiv-k^{\prime}-2 r k k^{\prime} \sigma^{2}-r k^{2}=0,
$$

where $k \equiv k\left(\sigma^{2}\right)$ and $k^{\prime} \equiv k^{\prime}\left(\sigma^{2}\right) \cdot{ }^{7}$ The effort cost parameter for the optimal technology follows from Equation (4) and it is $k\left(\sigma_{*}^{2}\right)$.

As the existence of a unique global maximum $\sigma_{*}^{2}$ will be useful for the comparative static analysis, we restrict the attention to the set of functions $k\left(\sigma^{2}\right)$ representing the technological relation between efficiency and riskiness that ensures this outcome. A unique maximum requires that the profit function $\pi^{*}$ in Equation (10) is increasing for $\sigma^{2}<\sigma_{*}^{2}$ and decreasing for $\sigma^{2}>\sigma_{*}^{2}$, that is $d \pi^{*} / d \sigma^{2} \gtreqless 0$ if $\sigma^{2} \lesseqgtr$ $\sigma_{*}^{2}$. Note that the sign of $d \pi^{*} / d \sigma^{2}$ is the same as the sign of the function $F\left(\sigma^{2}\right)$ in (12). Hence, from $F\left(\sigma_{*}^{2}\right)=0$, it follows that $F\left(\sigma^{2}\right)$ monotonically decreasing in $\sigma^{2}$ guarantees that $F\left(\sigma^{2}\right)>0$ when $\sigma^{2}<\sigma_{*}^{2}$ and $F\left(\sigma^{2}\right)<0$ when $\sigma^{2}>\sigma_{*}^{2} ;$ this in turn implies that $d \pi^{*} / d \sigma^{2} \gtreqless 0$ if $\sigma^{2} \lesseqgtr \sigma_{*}^{2}$ with $\sigma_{*}^{2}$ as the unique maximum. In other words, a unique maximum requires that

$$
d F\left(\sigma^{2}\right) / d \sigma^{2}=-4 r k k^{\prime}-2 r\left(k^{\prime}\right)^{2} \sigma^{2}-k^{\prime \prime}\left(1+2 r k \sigma^{2}\right)<0 .
$$

The first component of Equation (13) is positive (as $k^{\prime}<0$ ), the second is negative while the third one has the opposite sign of $k^{\prime \prime}$. Therefore, while $k\left(\sigma^{2}\right)$ can generally be concave or convex, a sufficient condition for Equation (13) to hold is that $k\left(\sigma^{2}\right)$ is sufficiently convex, i.e., that $k^{\prime \prime}$ is positive and large enough.

The existence of an interior solution $\sigma_{*}^{2} \in\left(0, \bar{\sigma}^{2}\right)$ to the equation $F\left(\sigma^{2}\right)=0$ in Equation (12) is guaranteed when $\lim _{\sigma^{2} \rightarrow 0} F\left(\sigma^{2}\right)>0$ and $\lim _{\sigma^{2} \rightarrow \bar{\sigma}^{2}} F\left(\sigma^{2}\right)<0$ given that $F\left(\sigma^{2}\right)$ is monotonically decreasing in $\sigma^{2}$. As the second component of Equation (12) is nonnegative, a sufficient condition that guarantees that $\lim _{\sigma^{2} \rightarrow 0} F\left(\sigma^{2}\right)>0$ is that $\lim _{\sigma^{2} \rightarrow 0}\left(-k^{\prime}\right)>\lim _{\sigma^{2} \rightarrow 0} r k^{2}$, i.e., that $\lim _{\sigma^{2} \rightarrow 0}\left(-k^{\prime} / r k^{2}\right)>1$, so that the sum of the first and third component of $F\left(\sigma^{2}\right)$ is strictly positive. ${ }^{8}$ From $\lim _{\sigma^{2} \rightarrow \bar{\sigma}^{2}} k^{\prime}=0$ and $\underline{k} \equiv k\left(\bar{\sigma}^{2}\right)>0$ follows that the first two components of Equation (12) tend to zero as $\sigma^{2}$ reaches the upper bound $\bar{\sigma}^{2}$; hence, $\lim _{\sigma^{2} \rightarrow \bar{\sigma}^{2}} F\left(\sigma^{2}\right)=-r \underline{k}^{2}<0$.

The following proposition summarizes these results.

Proposition 1. The principal chooses the technology with the variance $\sigma_{*}^{2}$ implicitly defined by Equation (12) and with the level of efficiency $k\left(\sigma_{*}^{2}\right)$ defined in Equation (4). The agent optimally chooses the action $e^{*}$ reported in Equation (7) and the coefficients of the linear payment scheme $\beta^{*}$ and $\alpha^{*}$ are defined by Equations (8) and (9) with $k \equiv k\left(\sigma_{*}^{2}\right)$ and $\sigma^{2} \equiv \sigma_{*}^{2}$ respectively.

\section{Agent's Risk Aversion and the Provision of Incentives}

In this section, we analyze how a variation in the agent's risk aversion affects the provision of incentives when, as, in our framework, such a variation also induces a change in the technology adopted. We then provide the conditions for a positive relationship between agent's risk aversion and incentives and finally illustrate our analysis in the case where the technology function $k\left(\sigma^{2}\right)$ has a specific functional form.

\subsection{The Effects of a Change of the Agent's Risk Aversion on Incentives}

We first note that a reduction in the agent's risk aversion increases the riskiness $\sigma_{*}^{2}$ as well as the efficiency $\left(k\left(\sigma_{*}^{2}\right)\right.$ goes down) of the technology chosen by the principal. Indeed, by applying the implicit function theorem to Equation (12), we obtain that

7 Note that the first two components of Equation (12) are positive, while the third one is negative.

8 For example, this is always the case if $k$ is bounded and $\lim _{\sigma^{2} \rightarrow 0}\left(-k^{\prime}\right)=+\infty$. 


$$
\frac{\partial \sigma_{*}^{2}}{\partial r}=-\frac{\partial F / \partial r}{\partial F / \partial \sigma^{2}}=-\frac{-2 r k k^{\prime} \sigma^{2}-k^{2}}{-4 r k k^{\prime}-2 r\left(k^{\prime}\right)^{2} \sigma^{2}-k^{\prime \prime}\left(1+2 r k \sigma^{2}\right)}<0,
$$

as the denominator is negative from the second order condition of maximization problem in Equation (10) and the numerator is also negative since the first order condition in Equation (12) implies that $-2 r k k^{\prime} \sigma^{2}-k^{2}=k^{\prime} / r<0$.

Now, we show that, while the reduction of the agent's risk aversion induces the principal to provide more incentives by increasing the agent's payment related to the output for any given technology adopted (it is immediate from Equation (8) that $\beta^{*}$ is decreasing in $r$ ), this result may no longer hold if the lower risk aversion of the agent leads the principal to change the technology employed (i.e., its efficiency and riskiness). In this case, the characteristics of the new technology may affects the optimal provision of incentives in ways that counterbalance the former (standard) effect.

The total effect of a reduction of the agent's risk aversion on the optimal share $\beta^{*}$ of output paid to the agent is obtained by total differentiation of Equation (8), which gives

$$
\frac{d \beta^{*}}{d r}=\underbrace{\frac{\partial \beta^{*}}{\partial r}}_{\text {direct effect (-) }}+\underbrace{\begin{array}{c}
\frac{\partial \beta^{*}}{\partial \sigma^{2}} \frac{\partial \sigma^{2}}{\partial r} \\
(-)(-)
\end{array}}_{\text {indirect riskiness effect }(+)}+\underbrace{\left.\begin{array}{c}
\frac{\partial \beta^{*}}{\partial k} \frac{\partial k}{\partial \sigma^{2}} \frac{\partial \sigma^{2}}{\partial r} \\
(-)
\end{array}\right)}_{\text {indirect efficiency effect (-) }}
$$

Let us now analyze each of these effects generated by a decrease in agent's risk aversion on $\beta^{*}$ in detail. The first component in Equation (15) represents the (standard) direct effect of a reduction of $r$ on $\beta^{*}$, namely the effect on $\beta^{*}$ if the same technology is employed. This component is equal to

$$
\frac{\partial \beta^{*}}{\partial r}=-\frac{k \sigma^{2}}{\left(1+r k \sigma^{2}\right)^{2}}
$$

and it is always negative as a lower agent's risk aversion makes it optimal for the principal to give more incentives and less insurance to the agent, which requires increasing the payment related to output (i.e., $\left.\beta^{*}\right)$.

The other two components in Equation (15) represent the indirect effect of the reduction of $r$ on $\beta^{*}$, i.e., the effect caused by a change in the technology employed (following by the reduction of the agent's risk aversion) on the incentives provided by the principal to the agent. From Equation (14) we know that the new technology is characterized by a higher riskiness and a higher efficiency which generate two opposing effects on $\beta^{*}$.

The higher riskiness $\sigma_{*}^{2}$ of the new project adopted when the agent's risk aversion decreases makes it optimal the provision of more insurance and less incentives to the agent as

$$
\frac{\partial \beta^{*}}{\partial \sigma^{2}}=-\frac{r k}{\left(1+r k \sigma^{2}\right)^{2}}<0
$$

In other words, a reduction of $r$ increases the riskiness of the optimal technology $\left(\partial \sigma_{*}^{2} / \partial r<0\right.$, see Equation (14)) and this increases the payment $\beta^{*}$ related to output (see Equation (17)). We call this the riskiness effect and it has the opposite sign of the (standard) direct effect generated by the reduction of the agent's risk aversion $r$.

However, the new technology is also characterized by a higher efficiency (i.e., a lower cost of effort $k$ ), which makes it optimal an increase of incentives as

$$
\frac{\partial \beta^{*}}{\partial k}=-\frac{r \sigma^{2}}{\left(1+r k \sigma^{2}\right)^{2}}<0
$$


Specifically, a reduction of $r$ leads to the adoption of a project which has a lower $k$ (together with a higher $\sigma^{2}$ ), and this increases the payment $\beta^{*}$ related to output. We call this the efficiency effect and it has the same sign of the direct effect caused by a lower $r .^{9}$

Hence, the net indirect effect due to the adoption of a riskier and more efficient technology may in general lead to an increase or a decrease of incentives. In particular, the following three situations may arise.

(i) When the efficiency effect dominates the riskiness effect, the net indirect effect has the same sign of the (standard) direct effect. Therefore, a lower agent's risk aversion increases the incentives that the principal provides to the agent; in other words, $\beta^{*}$ increases as $r$ lowers because the additional indirect effect generated by the change of the technology employed on the payment scheme reinforces the standard effect.

(ii) When instead the riskiness effect prevails over the efficiency effect, the net indirect effect due to the change of the technology adopted (generated by a less risk averse agent) implies that the principal may find it optimal to reduce the incentives provided to the agent. As in this case the indirect effect has the opposite sign of the (standard) direct effect, the final effect on the compensation scheme depends on which of these two effects prevails. If the indirect effect is not strong enough to more than compensate the (standard) direct effect, then the final effect is the same of that one described at the previous Point (i).

(iii) If the indirect effect more than compensate the (standard) direct effect, then the principal will find it optimal to decrease the incentives provided to the agent by reducing the share $\beta^{*}$ of the agent's payment related to the output as $r$ goes down.

This latter case is the most interesting for our theory as it represents the situation where the change of technology adopted induces a variation in the sign of the relationship (relative to the standard theory) between the agent's risk aversion and the degree of incentives provided by the principal to the agent. As a condition for observing a positive (rather than a negative) relationship between risk aversion and incentives is the dominance of the riskiness effect on the efficiency effect, so that the indirect effect has the opposite sign of the standard direct effect, we now try to understand under what conditions this situation is more likely.

\subsection{Agent's Risk Aversion and Incentives: The Conditions for a Positive Link}

Let us first analyze the case where the net indirect effect has the same sign of the direct effect, so that $d \beta^{*} / d r$ in (15) is always negative and, therefore, a lower agent's risk aversion leads to more incentives (this is the case described above at Point (i)).

From the last two components in the right-hand side of Equation (15), we know that the indirect effect is negative if

$$
\frac{\partial \sigma^{2}}{\partial r}\left(\frac{\partial \beta^{*}}{\partial \sigma^{2}}+\frac{\partial \beta^{*}}{\partial k} \frac{\partial k}{\partial \sigma^{2}}\right) \leq 0
$$

As from Equation (14), $\partial \sigma^{2} / \partial r$ is always negative, it is immediate to see that the above inequality requires that

$$
\frac{\partial \beta^{*}}{\partial \sigma^{2}}+\frac{\partial \beta^{*}}{\partial k} k^{\prime} \geq 0
$$

where $k^{\prime} \equiv \partial k / \partial \sigma^{2}$. Using Equations (17) and (18), we obtain that the inequality in Equation (19) is satisfied when the following condition holds.

9 A straightforward comparison shows that, differently from our model, the endogenous matching models only consider the direct and the indirect riskiness effect (e.g., Serfes [10]), but not the indirect efficiency effect. Thus, whereas under positive assortative matching the riskiness effect is negative (since riskier principals attract more risk-averse agents), under negative assortative matching the indirect riskiness effect is positive (since now riskier principals are matched with less risk-averse agents) and the final effect of risk on incentives may, in this case, be ambiguous. 
Condition 1. The elasticity $E_{k \sigma}$ of the technology function $k\left(\sigma^{2}\right)$ with respect to the variance is weakly greater than 1, i.e.,

$$
E_{k \sigma} \equiv-k^{\prime} \frac{\sigma^{2}}{k} \geq 1
$$

To better understand the intuition behind the result leading to Condition 1, let us again recall that a necessary condition for observing a positive (rather than a negative) relationship between risk aversion and incentives is the dominance of the riskiness effect on the efficiency effect, so that the indirect effect goes in the opposite direction of the (standard) direct effect and may eventually prevail over the latter. Otherwise, the usual relationship between risk aversion and incentives described in Case (i) holds.

The reduction of the agent's risk aversion induces the principal to adopt a more efficient and riskier technology. The higher riskiness of the technology makes it optimal for the principal to reduce the incentives (and increase the insurance) to the agent by making the payment more related to output (this riskiness effect leads to a reduction of $\beta^{*}$ ). The fact that the technology is more efficient induces instead the principal to increase the incentives to the agent by making his payment more related to output (this efficiency effect increases $\beta^{*}$ ). Now, if the function $k\left(\sigma^{2}\right)$ is elastic, then, for any given increase in the riskiness $\sigma^{2}$ of the new technology employed (and therefore for any given size of the riskiness effect), the increase in the efficiency of the selected technology (going in the standard direction) will be large. In turn, this will make more likely that the efficiency effect dominates the riskiness effect. Condition 1 simply states that the required threshold on elasticity $E_{k \sigma}$ for this phenomenon to take place is 1 . When, conversely, the function $k\left(\sigma^{2}\right)$ is rigid (namely for $E_{k \sigma}<1$ ) and Condition 1 does not hold, an increase in the riskiness of the adopted technology implies that the efficiency effect may dominate the riskiness effect.

In other words, the elasticity of the technology function $E_{k \sigma}$ positively affects the size of the efficiency effect relative to the riskiness effect. Given that the latter effect needs to prevail over the former to reverse the standard relationship between agent's risk aversion and incentives, the elasticity $E_{k \sigma}$ needs to be relatively small (i.e., Condition 1 does not have to hold) for this to happen.

It is also important to emphasize that $E_{k \sigma}<1$ does not guarantee a positive relationship between the agent's degree of risk aversion and incentives as the (standard) direct effect could more than compensate the (net) indirect effect, i.e., the situation described at Point (ii) above could apply. However, when this does not happen and the riskiness effect is stronger than the sum of the direct effect and the efficiency effect (i.e., Case (iii) applies), a higher agent's risk aversion may lead to a reduction of the incentive provided by the principal (i.e., to a reduction of $\beta^{*}$ ). This is the case when $d \beta^{*} / d r$ in (15) is positive. By substituting Equations (14), (16), (17), and (18) into Equation (15) and rearranging terms, we obtain that $d \beta^{*} / d r>0$ occurs when the elasticity $E_{k \sigma}$ of the technology function is lower than a given threshold $\hat{E}_{k \sigma}$, i.e., if the following condition holds. ${ }^{10}$

Condition 2. The elasticity $E_{k \sigma}$ of the technology function $k\left(\sigma^{2}\right)$ with respect to the variance is smaller than a threshold $\hat{E}_{k \sigma}<1$, i.e.,

$$
E_{k \sigma} \equiv-k^{\prime} \frac{\sigma^{2}}{k}<1-\frac{2 r k k^{\prime}\left(\sigma^{2}\right)^{2}+k^{2} \sigma^{2}}{4 r^{2} k k^{\prime}+2 r^{2}\left(k^{\prime}\right)^{2} \sigma^{2}+r k^{\prime \prime}\left(1+2 r k \sigma^{2}\right)} \equiv \hat{E}_{k \sigma}
$$

The following proposition summarizes these results.

10 Note that the threshold $\hat{E}_{k \sigma}$ is not necessarily always positive. Clearly, when $\hat{E}_{k \sigma}<0$, Condition 2 can never be satisfied and there is always a negative relationship between the agent's degree of risk aversion and the incentives provided by the principal as in the standard principal-agent framework. 
Proposition 2. A reduction in the agent's risk aversion $r$ generates two effects on the optimal share of output $\beta^{*}$ paid to the agent. The direct effect always increases $\beta^{*}$, while the indirect effect due to the higher riskiness and efficiency of the new technology adopted can lead to an increase or a decrease of $\beta^{*}$. The following results may be obtained.

(i) When Condition 1 is satisfied, both the direct and indirect effects have the same sign and a lower agent's risk aversion $r$ unambiguously increase $\beta^{*}$ (i.e., $\left.\partial \beta^{*} / \partial r<0\right)$ as in the standard principal-agent model.

(ii) When Condition 1 does not hold, the total effect of $r$ on $\beta^{*}$ can either be negative or positive, depending on the magnitude of the direct and of the indirect effects.

(iii) When Condition 2 holds, the indirect effect have the opposite sign of the direct effect and larger size; therefore, a lower agent's risk aversion $r$ unambiguously decreases $\beta^{*}$ (i.e., $d \beta^{*} / d r>0$ ), which is an opposite result to the one usually obtained in the standard principal-agent model.

To further clarify this point in more detail, we consider below a specific functional form for the relationship between the cost parameter $k$ of the agent and the risk of the project expressed by $\sigma^{2}$.

\subsection{An Example}

We assume that the function representing the technological set $k\left(\sigma^{2}\right)$ has a specific functional form characterized by constant elasticity, i.e., $k=\left(\sigma^{2}\right)^{-\eta}$, with $\sigma^{2} \in(0, \infty)$ and $\eta \in(0,1 / 2)$. ${ }^{11}$ This means that $k$ is finite and positive for all $\sigma^{2}, k^{\prime}=-\eta k\left(\sigma^{2}\right)^{-1}<0$, and $k^{\prime \prime}=\eta(\eta+1) k\left(\sigma^{2}\right)^{-2}>0$.

The first order condition in Equation (12) of the principal's maximization problem can be rewritten as

$$
F\left(\sigma^{2}\right) \equiv \eta\left(\sigma^{2}\right)^{-1}-r(1-2 \eta)\left(\sigma^{2}\right)^{-\eta}=0
$$

which implies that the variance of the optimal technology is equal to

$$
\sigma_{*}^{2}=\left[\frac{\eta}{r(1-2 \eta)}\right]^{\frac{1}{1-\eta}}
$$

As we showed for the general case, the function $F\left(\sigma^{2}\right)$ has the same sign of $d \pi^{*} / d \sigma^{2}$. From Equation (20), we obtain that

$$
\frac{d F\left(\sigma^{2}\right)}{d \sigma^{2}}=-\eta\left(\sigma^{2}\right)^{-2}+r \eta(1-2 \eta)\left(\sigma^{2}\right)^{-\eta-1}
$$

which is negative for all $\sigma^{2}<[r(1-2 \eta)]^{-\frac{1}{1-\eta}} \equiv \bar{\sigma}^{2}$. The parameter $\eta<1 / 2$ implies that $\sigma_{*}^{2}<\bar{\sigma}^{2}$ and, therefore, that the function $F\left(\sigma^{2}\right)$ is decreasing at $\sigma^{2}=\sigma_{*}^{2}$. As there is no other point at which $F\left(\sigma^{2}\right)=0$ (from Equation (21), it is immediate that $\sigma_{*}^{2}$ is unique), it follows that $F\left(\sigma^{2}\right)>0$ for all $\sigma^{2}<\sigma_{*}^{2}$ and $F\left(\sigma^{2}\right)<0$ for all $\sigma^{2}>\sigma_{*}^{2}$. This in turn implies that $d \pi^{*} / d \sigma^{2} \gtreqless 0$ if $\sigma^{2} \lesseqgtr \sigma_{*}^{2}$ and therefore that $\sigma_{*}^{2}$ is the unique maximum.

From $\eta<1 / 2$, it follows that Condition 1 does not hold (as $E_{k \sigma}=\eta<1$ ) and the indirect effect is positive, i.e., the change of technology induced by the lower agent's risk aversion $r$ leads to a reduction of $\beta^{*}$ (the riskiness effect dominates the efficiency effect). This indirect effect opposes to the direct effect which instead pushes for an increase in $\beta^{*}$. The total effect of a reduction of $r$ on $\beta^{*}$ can be computed by substituting Equations (16)-(18) and $\partial \sigma_{*}^{2} / \partial r$ (which is obtained from Equation (21)) into Equation (15). This leads to $\partial \beta^{*} / \partial r=0$ which means that, in this special case, the direct and indirect effect of a change in $r$ on $\beta^{*}$ exactly offset each other and therefore that a reduction in the agent's risk aversion leaves the fraction of output paid to the agent unchanged.

11 As will be clear below, $\eta<1 / 2$ is necessary to obtain an interior solution. 
From Equation (20), we also obtain that

$$
\frac{\partial^{2} \pi}{\partial \sigma^{2} \partial r}=\eta\left(\sigma^{2}\right)^{\eta-1}+(2 \eta-1)
$$

which can be either positive or negative for $\eta<1 / 2$ (e.g., if $\sigma^{2}=1$, the expression is positive for $\eta>1 / 3$ and negative for $\eta<1 / 3$ ) and, therefore, is in general compatible with both positive and negative assortative matchings, as discussed in Footnote 9.

\section{Extensions and Discussion}

In this section, we address two issues. We first describe the role played by asymmetric information in explaining the choice of the principal and then we show that our results are robust to changes of the baseline setting by analyzing a framework where the higher return of riskier projects does not imply a reduction of the marginal cost of effort.

It is worth emphasizing that, in absence of asymmetric information between the principal and the agent, each project has a net return for the principal $\pi=y-c(e)-\delta=e-(1 / 2) k e^{2}-\delta+\varepsilon$ because the agent's payment is equal to his certainty equivalent plus the cost of effort, i.e., it is equal to $\delta+(1 / 2) k e^{2}$; the principal optimally fully insures the agent when there is no moral hazard. As more efficient technologies have a lower marginal cost of effort $k$, this implies a higher expected value of the net return of the project as this is given by $\mathbb{E}(\pi)=e-(1 / 2) k e^{2}-\delta$. At the same time, more efficient technologies are also riskier as projects with a lower marginal cost of effort $k$ are associated to a higher variance $\sigma^{2}$ of the shock; this implies a higher variance of the project's net return as this is equal to the variance of the shock, i.e., $\operatorname{Var}(\pi)=\operatorname{Var}(\varepsilon)=\sigma^{2}$. However, in this case, our problem has a trivial solution: the risk neutral principal adopts the most efficient technology, i.e., the one with the lowest marginal cost of effort $\underline{k} \equiv k\left(\bar{\sigma}^{2}\right)$ and the highest variance $\bar{\sigma}^{2}$. Indeed, one can easily verify that the optimal agent's effort is $e^{*}=1 / k$ and therefore the optimal net return is equal to $\pi^{*}=1 /(2 k)-\delta+\varepsilon$; as the principal is risk neutral, she finds it optimal choosing the most efficient technology with $\underline{k} \equiv k\left(\bar{\sigma}^{2}\right)$. This result comes from the fact that, in absence of asymmetric information, the agent's risk premium is equal to zero (because the principal optimally bears all risk and provides full insurance to the agent) and therefore the technology with the highest expected return is the most efficient one. When there is asymmetric information between the principal and the agent, the net return of the project is represented by the expected net profit $\pi^{*}$ of the (risk-neutral) principal reported in Equation (10). From the maximization problem in Equation (10), we know that $\pi^{*}$ is increasing for $\sigma^{2}<\sigma_{*}^{2}$ and decreasing for $\sigma^{2}>\sigma_{*}^{2}$. In this setting, the agent's risk premium becomes positive because the provision of incentives requires that the principal does not fully insure the agents who has to bear some risk. Such a risk premium is affected not only by the agent's degree of risk aversion but also by the technology employed and by the optimal incentive scheme, which in turn depends on the characteristics (in terms of efficiency and riskiness) of the technology. It is the variation of this risk premium with the project implemented that contributes to break the monotonic positive relationship between $\pi^{*}$ and $\sigma^{2}$ that characterizes the setting without asymmetric information and that leads to an interior solution for the optimal project (even if the principal, who chooses the technology, is risk neutral).

We now compare our model results with those obtained in a similar setting where the projects among which the principal can choose yield a return $y=e+\varepsilon$, with the shock $\varepsilon \sim N\left(\mu\left(\sigma^{2}\right), \sigma^{2}\right)$, and with $\mu^{\prime} \equiv \partial \mu\left(\sigma^{2}\right) / \partial \sigma^{2}>0$, namely higher error means are associated with higher variances. For simplicity, $k$ is now given and independent on $\sigma^{2}$ so that the agent's cost is not linked to the variance of the technology employed, i.e., $c(e)$ is still given by Equation (3). ${ }^{12}$

12 We thank an anonymous reviewer for pointing this quite natural and interesting extension of the model to our attention. 
It is easy to verify that, similar to the baseline model, the optimal share of output paid to the agent $\beta^{*}$ is still given by Equation (8) and that the net principal's profit is the same as the one reported in Equation (10) with the addition of the mean $\mu\left(\sigma^{2}\right)$ of the shock and the fact that $k$ is fixed rather than being a function of $\sigma^{2}$, i.e., the principal's maximization problem now reads

$$
\max _{\sigma^{2}} \pi^{*}=\mu\left(\sigma^{2}\right)+\frac{1}{2 k\left(1+r k \sigma^{2}\right)}-\delta .
$$

Thus, the optimal project is the one with the variance $\sigma_{*}^{2}$ that satisfies the following first order condition

$$
\frac{\partial \pi^{*}}{\partial \sigma^{2}}=\mu^{\prime}-\frac{r}{2\left(1+r k \sigma^{2}\right)^{2}}=0
$$

Since $\mu^{\prime}>0$, Equation (23) will normally lead to a unique maximum for the choice of the project. The solution of the condition in Equation (23) will be interior when the second order condition of the maximization problem is satisfied, i.e., when

$$
\frac{\partial^{2} \pi^{*}}{\partial\left(\sigma^{2}\right)^{2}}=\mu^{\prime \prime}+\frac{r^{2} k}{\left(1+r k \sigma^{2}\right)^{3}}<0,
$$

which generally holds when $\mu^{\prime \prime} \equiv \partial^{2} \mu\left(\sigma^{2}\right) / \partial\left(\sigma^{2}\right)^{2}$ is negative and large enough.

From the first order condition in Equation (23), one can easily derive the following relationship between the equilibrium project variance and the degree of agent's risk aversion:

$$
\frac{\partial \sigma_{*}^{2}}{\partial r}=\frac{1-r k \sigma^{2}}{2\left[\mu^{\prime \prime}\left(1+r k \sigma^{2}\right)^{3}+r^{2} k\right]} .
$$

The second order condition in Equation (24) implies that the denominator of Equation (25) is negative and, therefore, the sign of $\partial \sigma_{*}^{2} / \partial r$ is the same as the sign of the term $r k \sigma^{2}-1$. This means that $\partial \sigma_{*}^{2} / \partial r<0$ if $r k \sigma^{2}<1$, i.e., a reduction of the agent's risk aversion leads the principal to select a riskier project when the levels of risk aversion, project variance and marginal cost of effort are relatively low.

Since now $k$ is unrelated to the project's variance $\left(\partial k / \partial \sigma^{2}=0\right)$, from Equation (15), we observe that the indirect efficiency effect (represented by the third component) is absent. This implies that, in this new framework, if $r k \sigma^{2}<1$ so that $\partial \sigma_{*}^{2} / \partial r<0$, the indirect effect of $r$ on $\beta^{*}$ coincides with the indirect riskiness effect (which is the key new effect at work in the baseline framework). ${ }^{13}$ The absence of the indirect efficiency effect in this setting only works in the direction of making our conclusions more robust as such effect only reinforces the standard direct effect.

Again, if the indirect effect (represented by the second component in Equation (15)) more than compensate the (standard) direct effect (given by the first term in Equation (15)), the principal optimally chooses a riskier project and decreases the incentives provided to the agent by reducing the share $\beta^{*}$ of the agent's payment related to the output when the agent's risk aversion $r$ declines (as described in Point (iii) at the end of Section 4).

In other words, the analysis just presented shows that the indirect riskiness effect highlighted in the baseline framework (and that is at the heart of the contribution of this work) is present and could dominate even more frequently in a setting where the project's riskiness is related to the error mean of the technology employed rather than to the marginal cost of effort.

13 Note that $\beta^{*}$ and $\partial \beta^{*} / \partial \sigma^{2}<0$ are still given by Equations (8) and (17), respectively. 


\section{Conclusions}

We have shown that, in a principal-agent model with endogenous technology choice, the usual negative trade-off existing between the agent's risk aversion and optimal incentives does not necessarily hold and can, in some cases, be reversed. We have shown this result by using a modified version of Holmstrom and Milgrom's [1] framework where the principal can choose among different technologies where those with higher efficiency also have higher riskiness.

Our analysis highlights how, in such a framework, the reduction of the agent's risk aversion induces the adoption of a riskier technology that, in some cases, can revert the usual effects and lead to a reduction of the level of incentives provided to the agent. We have shown that such a result is more likely to occur when the link between the efficiency of the technology and its riskiness is relatively weak. Moreover, we have presented a special case where the increase in the riskiness of the technology is such that its effect on incentives counterbalances all the other effects leading to a neutrality between agent's risk aversion and the degree of incentives provided.

While this work has highlighted a new channel affecting the incentive scheme in principal-agent relationships, we think that additional work is needed to properly disentangle the relationship between the choice of technology (e.g., its degree of innovation and riskiness) and the amount of incentives provided by the firm. However, we leave this task to future research.

Acknowledgments: The authors would like to thank two anonymous referees, Antonio Ciccone and Alfonso Gambardella for useful comments and suggestions. The usual disclaimers apply.

Author Contributions: All authors contributed equally to this manuscript.

Conflicts of Interest: The authors declare no conflict of interest.

\section{References}

1. Holmstrom, B.; Milgrom, P. Aggregation and linearity in the provision of intertemporal incentives. Econometrica 1987, 55, 303-328.

2. Rao, C.; Hanumantha, H. Uncertainty, entrepreneurship, and sharecropping in India. J. Polit. Econ. 1971, 79, 578-595.

3. Allen, D.W.; Lueck, D. Risk preferences and the economics of contracts, Am. Econ. Rev. 1995, 85, 447-451.

4. Aggarwal, R.; Samwick, A. The other side of the trade-off: the impact of risk on executive compensation. J. Polit. Econ. 1999, 107, 65-105.

5. Core, J.; Guay, W. Estimating the value of employee stock option portfolios and their sensitivities to price and volatility. J. Account. Res. 2002, 40, 613-630.

6. Wulf, J. Authority, risk, and performance incentives: evidence from division manager positions inside firms. J. Ind. Econ. 2007, 55, 169-196.

7. Prendergast, C. The tenuous trade-off between risk and incentives. J. Polit. Econ. 2002, 110, 1071-1102.

8. Wright, D.J. The risk and incentives trade-off in the presence of heterogeneous managers. J. Econ. 2004, 83, 209-223.

9. Legros, P.; Newman, A.F. Beauty is a beast, frog is a prince: assortative matching with nontransferabilities. Econometrica 2007, 75, 1073-1102.

10. Serfes, K. Risk sharing vs. incentives: contract design under two-sided heterogeneity. Econ. Lett. 2005, 88, 343-349.

11. Serfes, K. Endogenous matching in a market with heterogeneous principals and agents. Int. J. Game Theory 2008, 36, 587-619.

12. Li, F; Ueda, M. Why do reputable agents work for safer firms? Financ. Res. Lett. 2009, 6, 2-12.

13. Ackerberg, D.; Botticini, M. Endogenous matching and the empirical determinants of contract form. J. Polit. Econ. 2002, 110, 564-591.

14. Mookherjee, D.; Ray, D. Contractual structure and wealth accumulation. Am. Econ. Rev. 2002, 92, 818-849. 
15. Barros, F.; Macho-Stadler, I. Competition for managers and market efficiency. J. Econ. Manag. Strategy 1998, 7, 89-103.

16. Dam, K.; Perez-Castrillo, D. The principal-agent matching market. Front. Theor. Econ. 2006, 2, doi:10.2202/1534-5963.1257.

(c)

(C) 2018 by the authors. Licensee MDPI, Basel, Switzerland. This article is an open access article distributed under the terms and conditions of the Creative Commons Attribution (CC BY) license (http://creativecommons.org/licenses/by/4.0/). 